Published in final edited form as:

Science. 2018 November 23; 362(6417): 949-952. doi:10.1126/science.aat8642.

\title{
Molecular to organismal chirality is induced by the conserved myosin 1D.
}

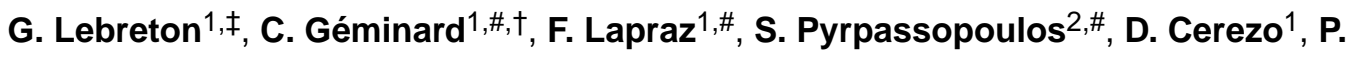 \\ Spéder ${ }^{1, \diamond}$, E. M. Ostap ${ }^{2}$, S. Noselli ${ }^{1, *}$ \\ 1 Université Côte D’Azur, CNRS, Inserm, Institut de Biologie Valrose, Nice, France. \\ ${ }^{2}$ Pennsylvania Muscle Institute and the Center for Engineering Mechanobiology, University of \\ Pennsylvania, Perelman School of Medicine, Phila-delphia, USA.
}

\begin{abstract}
Emergence of asymmetry from an initially symmetrical state is a universal transition in Nature. Living organisms show striking asymmetries at the molecular, cellular, tissular and organismal level. However, whether and how multilevel asymmetries are related remains unclear. Here, we show that Drosophila Myosin 1D (Myo1D) and Myosin 1C (Myo1C) are sufficient to generate de novo directional twisting of cells, single organs or the whole body in opposite directions. We show that directionality lies in the Myosins' motor domain and is swappable, and that Myo1D powers gliding of actin filaments in circular, counterclockwise paths in vitro. Altogether, our results reveal the molecular motor Myo1D as a chiral determinant, sufficient to break symmetry at all biological scales through chiral interaction with the actin cytoskeleton.
\end{abstract}

\section{One Sentence Summary:}

The Myosin1D molecular motor is a chiral determinant breaking symmetry at all biological scales.

\begin{abstract}
A fundamental feature of biological systems lies in their assembly from homochiral molecular components. Whether the macroscopic asymmetries of living organisms are directly related to their molecular chirality, as suggested by the 'F-molecule hypothesis' (1), remains an open question. The multiscale nature of asymmetries within organisms further raises the question of their origin (single vs. multiple determining events) and mode of propagation through different organization layers. We use Left-Right (LR) asymmetry establishment (2-5) to address these questions. The conserved myosin 1D(myo1D) gene is
\end{abstract}

\footnotetext{
"Correspondence to: noselli@unice.fr.

†Present addresses:

Centre de Biologie Intégrative, Université Paul Sabatier, 118 Route de Narbonne, 31062 TOULOUSE Cedex, France.

$\dagger$ Institut de Recherche en Cancérologie de Montpellier (IRCM), Inserm U1194, 208 avenue des Apothicaires, 34298 Montpellier cedex5.

Institut Pasteur, 25 rue du Dr Roux, 75015 Paris.

\#These authors contributed equally to this work

Author contributions: G.L. and S.N. designed the experiments. G.L., C.G., F.L. performed the experiments (except gliding assays), with the help of D.C. P.S. constructed the chimeras. S.P. performed and S.P. and E.M.O. designed and analyzed the gliding assays.

G.L., E.M.O. and S.N. wrote the manuscript with input from all of the authors.

Competing interests: Authors declare no competing interests.

Data and materials availability: All data is available in the main text or the supplementary materials.
} 
a unique situs inversus gene, essential for Dextral looping of all native LR organs in Drosophila (6-9). Absence of myo1D leads to inverted LR morphogenesis with organs adopting a Sinistral orientation. Drosophila has several independent, tissue-specific LR organizers in which myo1D is expressed and necessary (10-14).

To determine whether myo1D may be sufficient to drive LR asymmetry, we ectopically expressed the protein in different naive tissues, (i.e., devoid of LR asymmetry). Strikingly, Myo1D expression in the larval epidermis induces dextral twisting of the whole larval body (Fig. 1A,B). This remarkable phenotype is $100 \%$ penetrant and is specific to Myo1D (Fig. S1A-E). The larval body can rotate up to $180^{\circ}$, flipping the mouth parts towards the dorsal side of the larvae (Fig. 1B) and misaligning adjacent denticle belts by $17.4^{\circ}\left(0^{\circ}\right.$ in WT) (Fig. S1F,G,I). Of note, twisted posture alters locomotion behavior, the larvae moving through directional barrel rolling rather than normal crawling (Movie S1). Dextral twisting can also be induced in pupae and adult abdomens (Fig. S2A-D).

To test whether myo1D can induce asymmetry at the single organ level, we ectopically expressed it in tracheal precursors. In this condition, the whole trachea undergoes dramatic Dextral twisting (see Materials \& Methods), adopting a spiraling ribbon shape with multiple turns, instead of the smooth and linear conformation of WT trachea (Fig.1C,D; Fig. S3). We next investigated twisting at the cellular level, through quantification of the geometry and polarity of epidermal cells expressing Myo1D ectopically. In control conditions, cell membrane orientation shows a Gaussian distribution centered on $0^{\circ}$ (i.e., cells are perpendicular to the AP axis) (Fig. 1E). In contrast, Myo1D-expressing cells show elongation (Fig. S4) and a clear shift in membrane orientation towards one side (Fig. 1F), indicating that Myo1D also induces directional polarization at the cell level.

Head/motor, neck and tail domains are essential for normal Myo1D function (15). To assess their requirement for myo1D gain-of-function (GOF) phenotypes, we expressed pointmutated or truncated Myo1D proteins (15). Results show that integrity of the protein is essential, all domains being required (Fig. 1G). Interestingly, point mutations in actin-or ATP-binding sites indicate that Myo1D actin-based motor activity is crucial for its function in establishing both native (15) and de novo LR asymmetry (Fig. 1).

To test Myo1D specificity in generating de novo asymmetry, we ectopically expressed seven other Drosophila Myosins (Fig. 2A-C; Fig. S5A-G). Only Myo1C (16) overexpression led to twisted larvae (Fig. 2C). Remarkably, Myo1C-induced twisting is opposite (Sinistral) to that of Myo1D (Fig. 2A-C) and less pronounced ( $90^{\circ}$ vs. $180^{\circ}$ for Myo1D), with a denticle belt angle of $-9.9^{\circ}$ instead of $17.4^{\circ}$ for Myo1D (Fig. S1F,H,I). Myo1C also induces twisting of trachea (Fig. 2E,F), pupae, adults (Fig. S2E,F), as well as spiraling locomotion (Movie S2), all with sinistral orientation. Additionally, Myo1C-expressing cells show opposite lateralized polarity to Myo1D ones (Fig. 2G-H). Finally, Myo1C requires the same domain integrity as Myo1D for sinistral twisting (Fig. 2I), in particular a fully functional motor domain. Interestingly, we found that both Myosins are antagonistic, cancelling out each other's GOF phenotype (Fig. 2B-D; Fig. S2G,H), reminiscent of previous data showing that Myo1C can antagonize Myo1D in native LR organs $(6,17)$. 
The fact that two paralogous Myosins can polarize the whole larvae in opposite ways provides a unique situation to determine the molecular basis of directionality. Thus, we performed a structure-function analysis swapping the head, neck and tail domains from each protein (Fig. 3). Expression of each chimera in the larvae showed that the determinant for directionality in these myosins lies in their motor/head domain (Fig. 3). Keeping the neck and tail domains from the same protein increases protein activity, while swapping the sole neck reduces it dramatically (Fig. 3), suggesting an important coupling between head and neck for full myosin activity. Myosin head domains are known to directly interact with actin filaments. We found that reducing Profilin-Chickadee protein expression led to suppression or strong reduction of the body twisting phenotypes induced by either Myosin (Fig. S6A-G), pointing to actin formation/dynamics for Myosins chiral activity. Next, we assessed the ability of myosins to produce motion of actin filaments while bound to fluid supported lipid bilayers in vitro (18). Both Myosins bind to PI(4,5)P2 (Fig. S7) and power actin filament motility on supported lipid bilayers (Movie S3), with Myo1D having a speed 1.7 fold faster than Myo1C (Table S1). Strikingly, Myo1D powered clear counterclockwise, circular motility of actin, while Myo1C did not show any turning bias in these conditions (Fig. 4A,B, Table S1). Circular motility persisted when Myo1D was attached to a fluid bilayer via a biotin-streptavidin linkage confirming the finding that asymmetric motility is a property of the motor domain. Importantly, mixing the two proteins and increasing Myo1C/Myo1D ratio reduces circular F-actin movement, thus recapitulating the Myo1C antagonism towards Myo1D observed in vivo for normal and de novo LR asymmetry (Fig. 2D; Fig. 4C) $(6,17)$. Altogether, these results reveal a chiral interaction between Myo1D and F-actin in vitro, providing evidence for a molecular origin of LR asymmetry induced by this Myosin at the cell, organ and whole-body level.

In conclusion, Myo1D represents a unique chiral determinant, necessary for native handedness and sufficient to create de novo LR asymmetry at all scales (from molecular to behavioral), with chiral information being encoded within the motor domain itself. We propose that the multiscale property of Myo1D emerges from its molecular chiral interaction with F-actin (Fig. 4D). This model is in accordance with the so-called 'F-molecule' model for LR asymmetry establishment (1), predicting that chiral factors (in particular, molecular motors) set axis direction at the molecular level through vector information then propagating across organization scales.

Myo1D's ability to generate de novo asymmetry has two important meanings. First, it indicates that some tissues are competent but lack chiral determinant activity, Myo1D exposing an intrinsic yet only partly 'unfolded' polarized LR axis. Second, the large-scale changes in organ/body shape and posture/behavior triggered by simple misregulation of Myo1D fits the fundamental property of so-called 'toolkit-genes' proposed to control morphological evolution (19). Hence, our findings provide clues to understand the origin of torsion in evolution, proposed to rely on a single genetic event ('macromutation') for gastropod's $180^{\circ}$ torsion (20). Accordingly, recent results have shown the essential role of actin regulators in snail coiling $(21,22)$. 


\section{Supplementary Material}

Refer to Web version on PubMed Central for supplementary material.

\section{Acknowledgments:}

We wish to thank Kenji Matsuno, Jordi Casanova and Tianming Lin for reagents; the Bloomington Drosophila Stock Center, the National Institute of Genetics Fly (NIG-Fly) and Vienna Drosophila RNAi Center (VDRC) for providing Drosophila fly lines; the iBV PRISM platform; Melanie Gettings (Getting Published) for comments and members of the SN laboratory for discussions.

Funding: FL is supported by Agence Nationale pour la Recherche (ANR; ANR-13-BSV2-0006) and Université Côte d'Azur (UCA); GLB by ANR (ANR-13-BSV2-0006). EMO is supported by NIH Grant R37GM057247. Work in SN laboratory is supported by ANR (ANR-13-BSV2-0006; ANR-17-CE13-0024), UCA, Centre National pour la Recherche Scientifique (CNRS), Institut National pour la Recherche Médicale (Inserm), and the LABEX SIGNALIFE (ANR-11-LABX-0028-01).

\section{References}

1. Brown NA, Wolpert L, The development of handedness in left/right asymmetry. Development. 109, 1-9 (1990). [PubMed: 2209459]

2. Blum M, Feistel K, Thumberger T, Schweickert A, The evolution and conservation of left-right patterning mechanisms. Development. 141, 1603-13 (2014). [PubMed: 24715452]

3. Coutelis J-B, González-Morales N, Géminard C, Noselli S, Diversity and convergence in the mechanisms establishing L/R asymmetry in metazoa. EMBO Rep. 15, 926-37 (2014). [PubMed: 25150102]

4. Nakamura T, Hamada H, Left-right patterning: conserved and divergent mechanisms. Development. 139, 3257-3262 (2012). [PubMed: 22912409]

5. Raya Á, Belmonte JCI, Left-right asymmetry in the vertebrate embryo: from early information to higher-level integration. Nat. Rev. Genet. 7, 283-293 (2006). [PubMed: 16543932]

6. Hozumi S et al., An unconventional myosin in Drosophila reverses the default handedness in visceral organs. Nature. 440, 798-802 (2006). [PubMed: 16598258]

7. Spéder P, Adám G, Noselli S, Type ID unconventional myosin controls left-right asymmetry in Drosophila. Nature. 440, 803-7 (2006). [PubMed: 16598259]

8. Tingler M et al., A Conserved Role of the Unconventional Myosin 1d in Laterality Determination. Curr. Biol. (2018), doi:10.1016/j.cub.2018.01.075.

9. Juan T et al., Myosin1D is an evolutionarily conserved regulator of animal left-right asymmetry. Nat. Commun. 9 (2018), doi:10.1038/s41467-018-04284-8.

10. Coutelis JB, Petzoldt AG, Speder P, Suzanne M, Noselli S, Left-right asymmetry in Drosophila. Semin. Cell Dev. Biol. 19, 252-262 (2008). [PubMed: 18328746]

11. Geminard C, Gonzalez-Morales N, Coutelis JB, Noselli S, The myosin ID pathway and left-right asymmetry in Drosophila. Genesis. 52, 471-480 (2014). [PubMed: 24585718]

12. Spéder P, Noselli S, Left-right asymmetry: class I myosins show the direction. Curr. Opin. Cell Biol. 19, 82-87 (2007). [PubMed: 17174542]

13. González-Morales N et al., The Atypical Cadherin Dachsous Controls Left-Right Asymmetry in Drosophila. Dev. Cell. 33, 675-689 (2015). [PubMed: 26073018]

14. Suzanne M et al., Coupling of Apoptosis and L/R Patterning Controls Stepwise Organ Looping. Curr. Biol. 20, 1773-1778 (2010). [PubMed: 20832313]

15. Hozumi S et al., Head region of unconventional myosin I family members is responsible for the organ-specificity of their roles in left-right polarity in Drosophila. Dev. Dyn. 237, 3528-3537 (2008). [PubMed: 18521948]

16. Morgan NS, Skovronsky DM, Artavanis-Tsakonas S, Mooseker MS, The molecular cloning and characterization of Drosophila melanogaster myosin-IA and myosin-IB. J. Mol. Biol. 239, 347356 (1994). [PubMed: 8201616] 
17. Petzoldt AG et al., DE-Cadherin regulates unconventional Myosin ID and Myosin IC in Drosophila left-right asymmetry establishment. Development. 139, 1874-1884 (2012). [PubMed: 22491943]

18. Pyrpassopoulos S, Feeser EA, Mazerik JN, Tyska MJ, Ostap EM, Membrane-Bound Myo1c Powers Asymmetric Motility of Actin Filaments. Curr. Biol. 22, 1688-1692 (2012). [PubMed: 22863317]

19. Carroll SB, Evo-Devo and an Expanding Evolutionary Synthesis: A Genetic Theory of Morphological Evolution. Cell. 134, 25-36 (2008). [PubMed: 18614008]

20. Ponder WF, Lindberg DR, Towards a phylogeny of gastropod mollusc: An analysis using morphological characters. Zool. J. Linn. Soc. 119, 83-265 (1997).

21. Kuroda R et al., Diaphanous gene mutation affects spiral cleavage and chirality in snails. Sci. Rep. 6, 34809 (2016). [PubMed: 27708420]

22. Davison A et al., Formin Is Associated with Left-Right Asymmetry in the Pond Snail and the Frog. Curr. Biol, 1-7 (2016).

23. Brand AH, Perrimon N, Targeted gene expression as a means of altering cell fates and generating dominant phenotypes. Development. 118, 401-15 (1993). [PubMed: 8223268]

24. Heller D et al., EpiTools: An Open-Source Image Analysis Toolkit for Quantifying Epithelial Growth Dynamics. Dev. Cell. 36, 103-116 (2016). [PubMed: 26766446]

25. Tenenbaum CM, Gavis ER, Removal of \&lt;em\&gt;Drosophila\&lt;/em\&gt; Muscle Tissue from Larval Fillets for Immunofluorescence Analysis of Sensory Neurons and Epidermal Cells. J. Vis. Exp. (2016), doi:10.3791/54670.

26. El Mezgueldi M, Tang N, Rosenfeld SS, The kinetic mechanism of myole (human myosin-IC). J. Biol. Chem. 277, 21514-21521 (2002). [PubMed: 11940582]

27. Pyrpassopoulos S, Shuman H, Michael Ostap E, Single-molecule adhesion forces and attachment lifetimes of Myosin-I phosphoinositide interactions. Biophys. J. 99, 3916-3922 (2010). [PubMed: 21156133]

28. Spudich JA, Watt S, The regulation of rabbit skeletal muscle contraction. I. Biochemical studies of the interaction of the tropomyosin-troponin complex with actin and the proteolytic fragments of myosin. J. Biol. Chem. 246, 4866-4871 (1971). [PubMed: 4254541]

29. Putkey JA, Slaughter GR, Means AR, Bacterial expression and characterization of proteins derived from the chicken calmodulin cDNA and a calmodulin processed gene. J. Biol. Chem. 260, 47044712 (1985). [PubMed: 2985564]

30. Abràmofff MD, Magalhães PJ, Ram SJ, Image processing with ImageJ Part II. Biophotonics Int. 11, 36-43 (2005). 

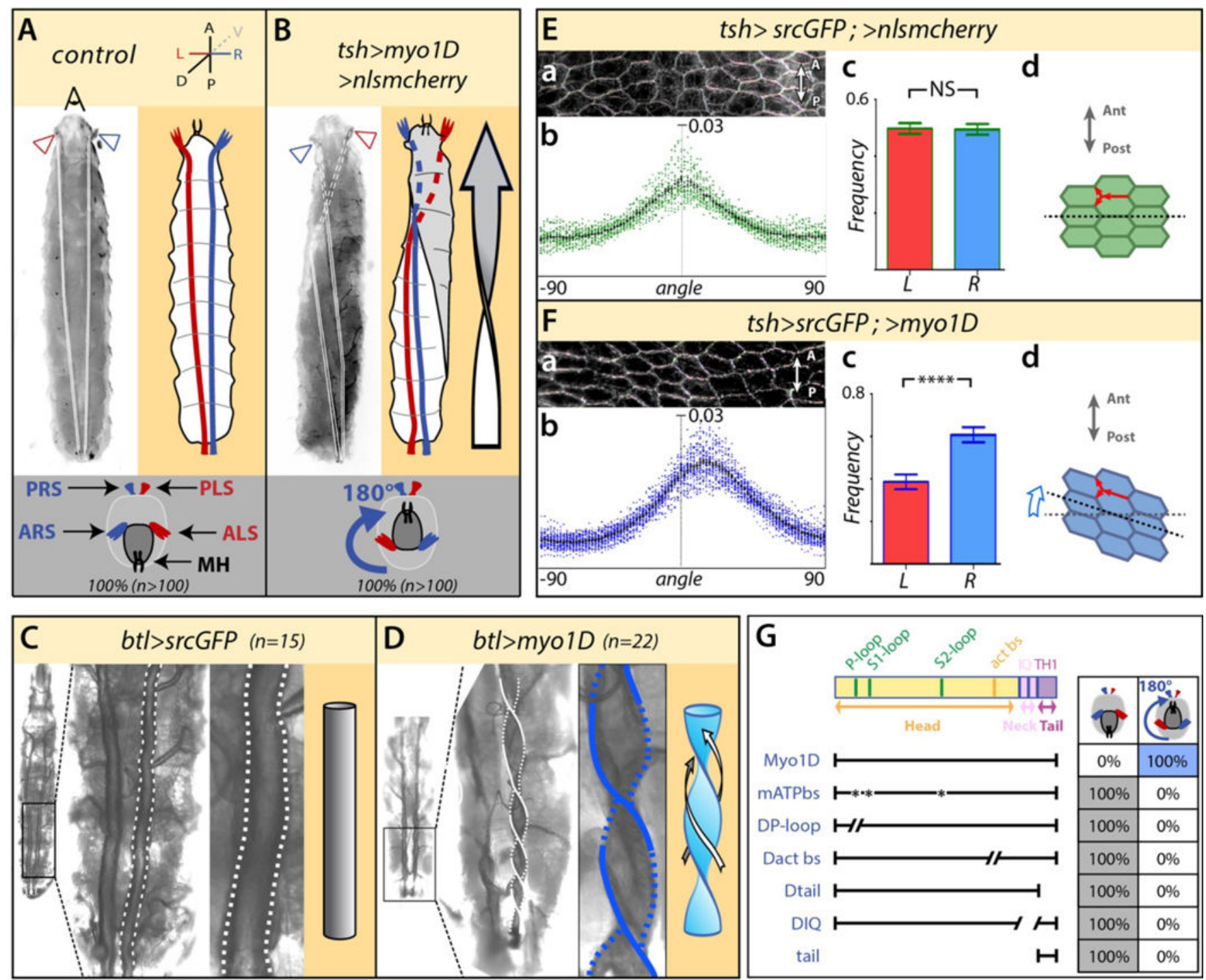

Fig. 1: Myosin 1D induces de novo Left-Right Asymmetry

A, Control larvae (white, left panel; schematized, right panel) showing perfect bilateral symmetry. Trachea highlighted, with anterior tracheal spiracles indicated by a red (left) or blue (right) open triangle. Bottom panel: front view of the larvae (ventral down, dorsal up); $\mathrm{MH}$, mouth-hooks; ALS, anterior left spiracle; ARL, anterior right spiracle; PLS, posterior left spiracle; PRS, posterior right spiracle. Left and right spiracles shown in red and blue, respectively.

B, myo1D expression in the epidermis ( $t s h>m y o 1 D)$ is sufficient to induce Dextral twisting of the whole larval body by $180^{\circ}$.

C, Linear morphology of a control ( $b t I>s r c G F P)$ third instar larvae trachea.

D, myo1D expression in the tracheae ( $b t 1>$ myolD) induces their Dextral twisting with multiple loops.

Ea-Ed, Fa-Fd, Morphometric analysis of the larval epidermis of wild type (Ea) and myo1D-expressing epidermal cells $(\mathbf{F a})$. Graphic plot showing distribution of cellular angles 
relative to the AP axis $(\mathbf{E b}, \mathbf{F b})$ and plot of the sum of rightward $\left(-90^{\circ}\right.$ to $\left.0^{\circ}: \mathrm{R}\right)$ against leftward $\left(0^{\circ}\right.$ to $\left.90^{\circ}: \mathrm{L}\right)$-oriented angles $(\mathbf{E c}, \mathbf{F c})$, showing that myo1D overexpression induces polarized reorganization of epithelial cells towards Dextral (Ed,Fd). $\mathrm{n}=24$ for $t s h>s r c$ GFP>nls-mCherry; $\mathrm{n}=39$ for $t s h>m y o 1 D-R N A i>s r c-G F P$. Error bars indicate SD. ${ }^{* * * *}$, $\mathrm{p}<0.0001$; NS, non-significant.

G, Structure-function analysis of Myo1D twisting activity. Integrity of the protein as well as its ATP and actin-binding sites are essential for its function. $\mathrm{n}=25$ for each. 

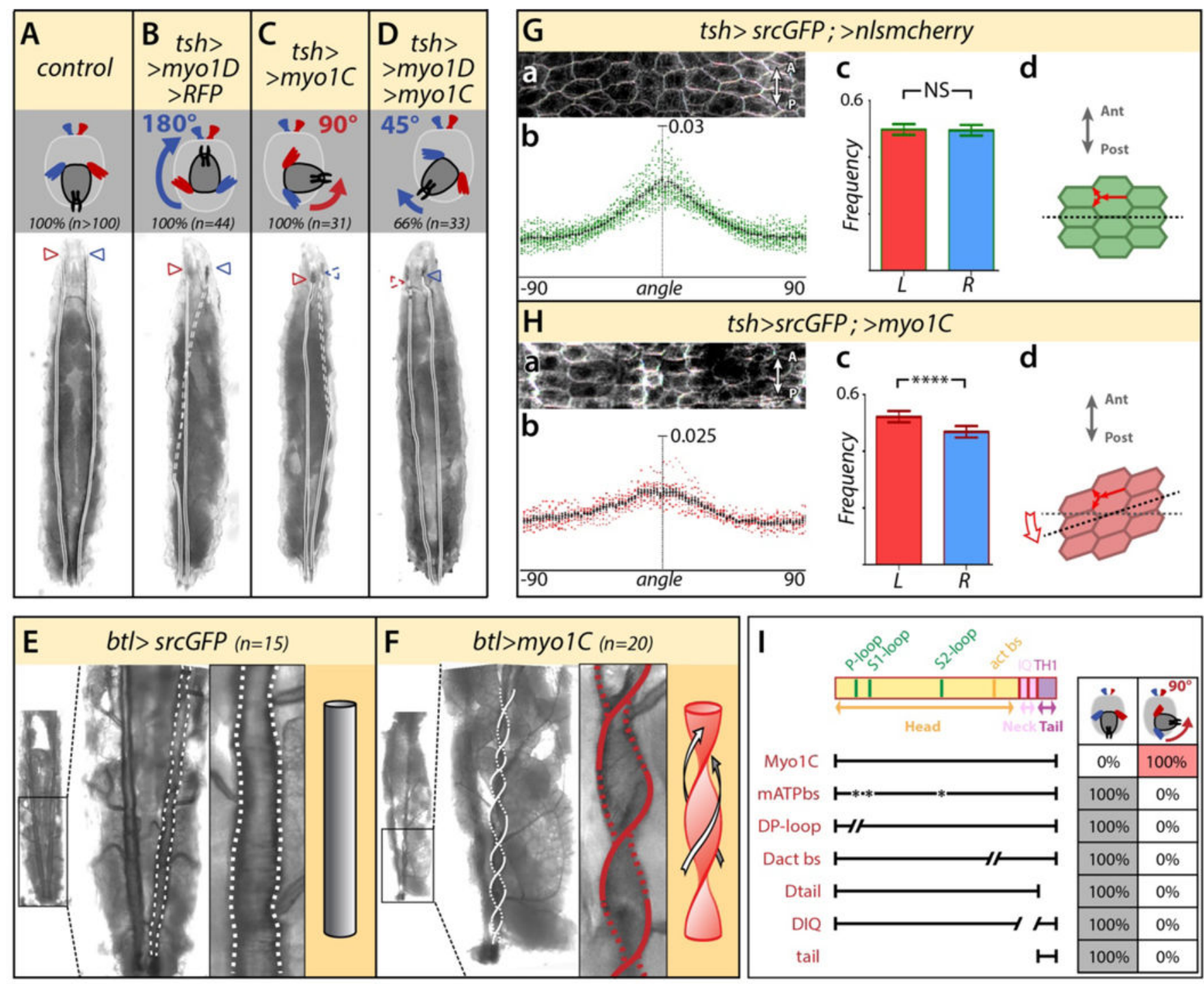

Fig. 2: Myosin 1C is a sinistral Myosin antagonist to Myo1D

A-D, Control (white) larvae (A), and larval twisting phenotype induced by myo1D(B), myolC $(\mathbf{C})$ overexpression or myolD and myolC co-expression (D).

E, Linear morphology of a control ( $b t />s r c G F P)$ third instar larvae trachea.

F, myolc expression in the tracheae (btl>myo1C) induces their Sinistral twisting.

Ga-Gd, Ha-Hd, Morphometric analysis of the larval epidermis of wild type (Ga) and myo1C-expressing epidermal cells (Ha). Graphic plot showing distribution of cellular angles relative to the AP axis $(\mathbf{G b}, \mathbf{H b})$ and plot of the sum of rightward $\left(-90^{\circ}\right.$ to $\left.0^{\circ}: \mathrm{R}\right)$ against leftward $\left(0^{\circ}\right.$ to $\left.90^{\circ}: \mathrm{L}\right)$-oriented angles $(\mathbf{G c}, \mathbf{H c})$, showing that myolCoverexpression induces polarized reorganization of epithelial cells towards Sinistral (Gd,Hd). n=24 for $t s h>s r c$ GFP>nls-mCherry; $\mathrm{n}=12$ for $t s h>m y o 1 C-R N A i>s r c-G F P$. Error bars indicate SD. ****, $\mathrm{p}<0.0001$; NS, non-significant.

I, Structure-function analysis of Myo1C twisting activity. Integrity of the protein as well as its ATP and actin binding sites are essential for its function. $\mathrm{n}=25$ for each. 


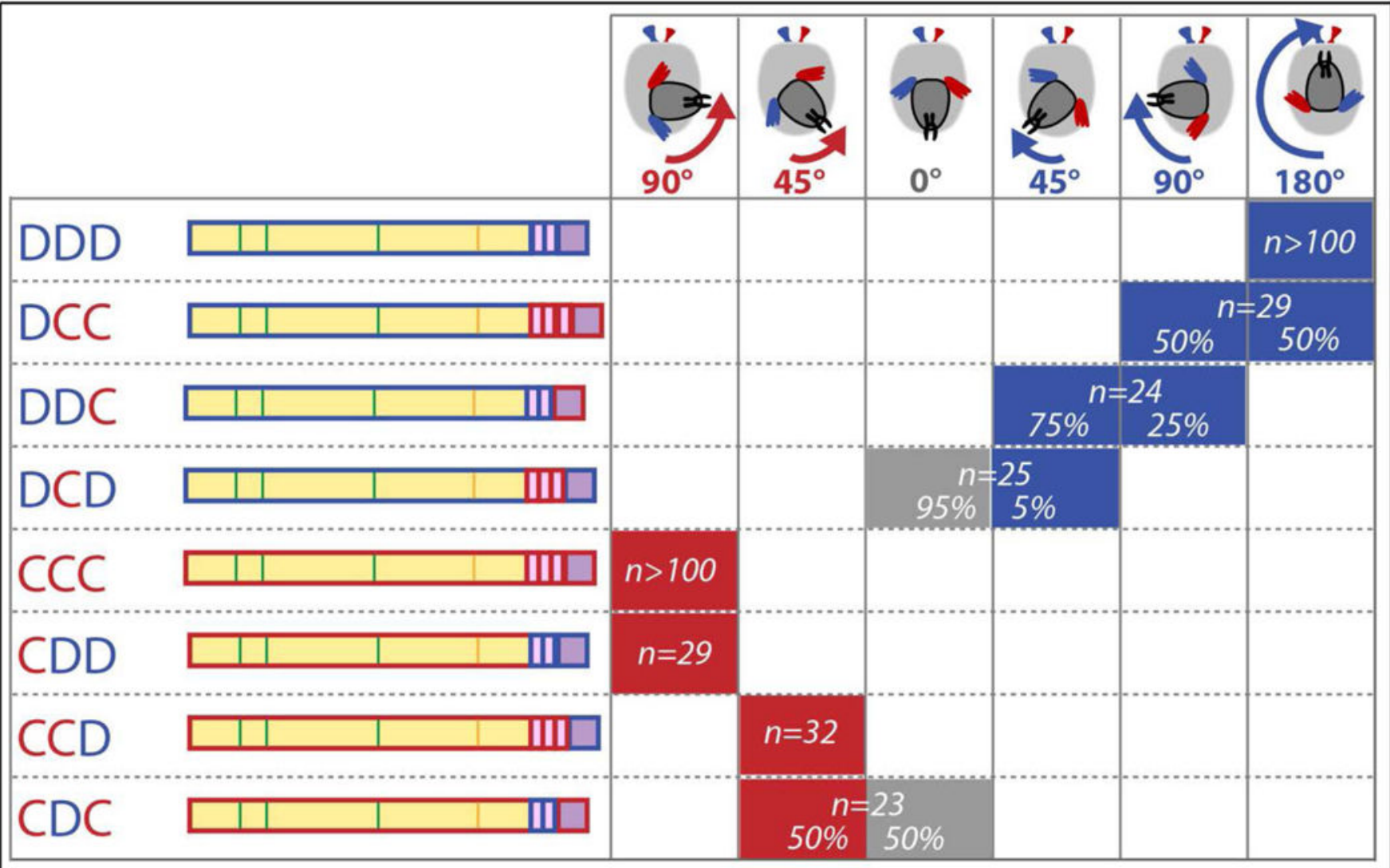

Fig. 3: The motor domain confers Myo1D and Myo1C chirality

A, Twisting activity of all Myo1D-Myo1C chimeras swapping Head/Motor, Neck and Tail domains. The head domain provides directionality to the proteins. Domains designated D and $\mathrm{C}$ pertain to Myo1D and Myo1C, respectively. 
A

B
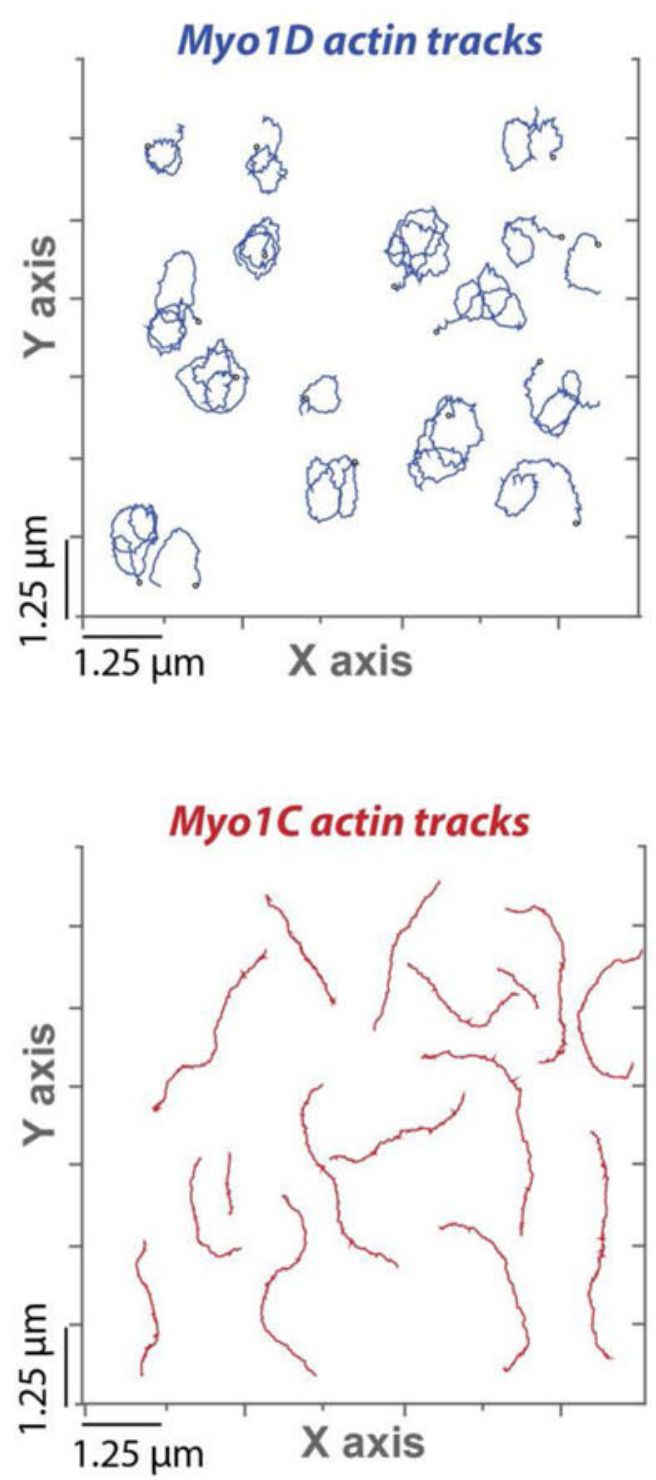

C

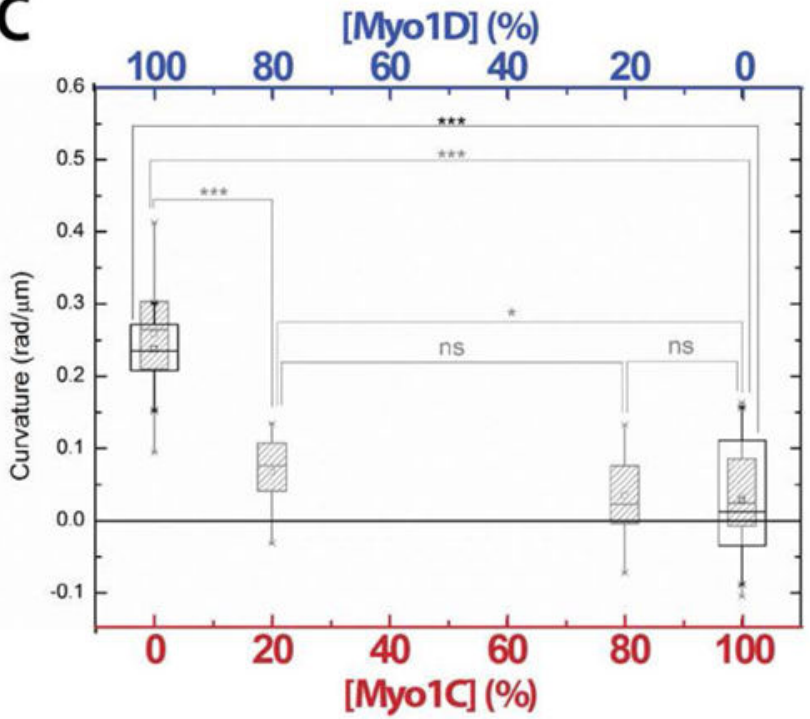

D
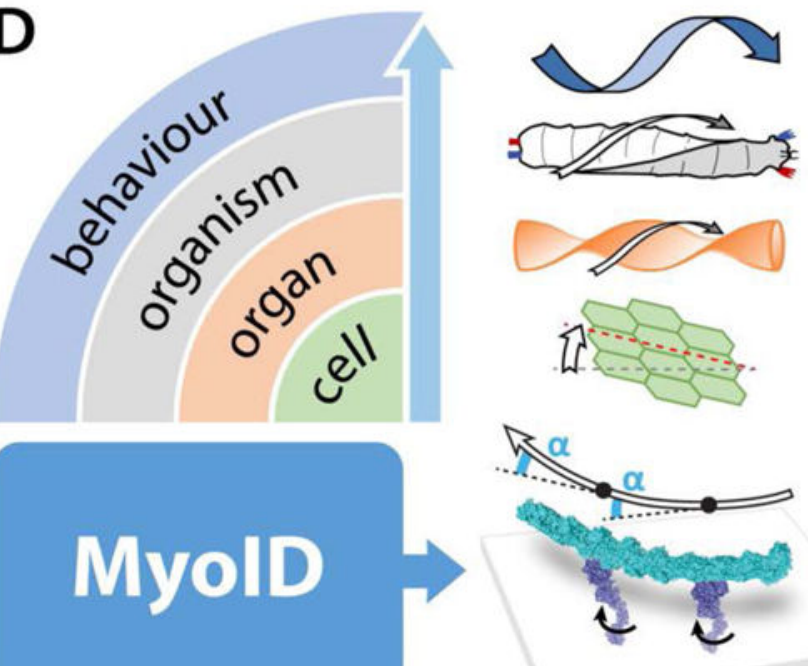

Fig. 4: Chiral interaction between Myo1D and F-actin in vitro

A,B, Representative tracks (track origin indicated by open circle) of actin filaments from actin gliding assays on 2\% PI(4,5)P2 - 98\% DOPC supporting lipid bilayers for Myo1D (A) and Myo1C (B).

C, Curvature of actin filament tracks on $2 \% \mathrm{PI}(4,5) \mathrm{P} 2-98 \%$ DOPC (black boxes) and $2 \%$ biotinPE - 98\% DOPC (grey boxes) supported lipid bilayers for different relative Myo1D and Myo1C concentrations.

D, Model of Myo1D chiral activity across multiple organization scales. Bottom cartoon represents (cyan) actin filament gliding powered by (purple) Myo1D on (grey) lipid bilayers. The white arrow indicates actin gliding direction. For most myosins, power stroke occurs in the direction of the actin filament axis. Mechanisms for actin turning (angle a) include (a) myosin lever-arm translation no longer along the long-axis of the actin filament, (b) myosin 
lever arm circular rotation during the power stroke (arrows), or (c) myosin-induced actin conformational change that results in actin filament leftward bending. 